\title{
Arthroscopic treatment of synovial chondromatosis of the proximal \\ tibiofibular joint: A case report
}

\author{
Sang Jin Lee
}

Department of Orthopedic Surgery, Haeundae Paik Hospital, College of Medicine, Inje University, 875 Haeundaero, Haeundaegu, Busan, Korea

\section{CASE STUDY}

Please cite this paper as: Lee SJ. Arthroscopic treatment of synovial chondromatosis of the proximal tibiofibular joint: $A$ case report. AMJ 2018;11(12):573-577.

https://doi.org/10.21767/AMJ.2018.3547

\section{Corresponding Author:}

Sang Jin Lee

Department of Orthopedic Surgery, Haeundae Paik Hospital, College of Medicine, Inje University, 875 Haeundaero, Haeundaegu, Busan, Korea

Email: sjinnie@paik.ac.kr

\section{ABSTRACT}

A 14-year-old girl presented with pain during the motion of knee joint. Multiple loose bodies were detected in proximal tibiofibular joint area in simple radiography. MRI demonstrated multiple small calcifications in the cystic lesion which was located around the popliteus muscle from the proximal area of fibular head to the tibial attachment area of popliteus muscle. Loose bodies were excised successfully with only arthroscopic approach through popliteal hiatus. To our knowledge, arthroscopic excision of synovial chondromatosis of the proximal tibiofibular joint has not been reported.

\section{Key Words}

Synovial chondromatosis, tibiofibular joint, arthroscopic treatment

\section{Implications for Practice:}

\section{What is known about this subject?}

Synovial chondromatosis in the proximal tibiofibular joint has been removed by arthrotomy or open debridement.

\section{What new information is offered in this case study?}

Removing the loose bodies around the popliteal muscle and the proximal tibiofibular joint was performed successfully through the popliteal hiatus using only arthroscopic approach.

3. What are the implications for research, policy, or practice?

Arthroscopic approach should be considered in the treatment for synovial chondromatosis around the popliteal muscle and the proximal tibiofibular joint.

\section{Background}

Synovial chondromatosis is an unusual disease characterized by the formation of multiple cartilaginous nodules that result from proliferative metaplasia of the mesenchymal cells in the synovial membrane of joint, tendon sheath and bursae. ${ }^{1}$ It is most often diagnosed in the male patients during the third to fifth decades of life and most commonly involves the knee joint. ${ }^{2,3}$ Synovial chondromatosis is classified as intra-articular and extraarticular. The intra-articular synovial chondromatosis may be either localized or generalized. The disease predominantly occurs in the anterior compartment of the knee joint such as suprapatellar pouch, infrapatellar fat pad and the space between anterior cruciate ligament and infrapatellar fat pad. ${ }^{4}$

Cases of synovial chondromatosis in the posterior compartment of knee joint have been reported rarely. ${ }^{4-9}$ Furthermore, arthroscopic treatment of synovial chondromatosis of the proximal tibiofibular joint has not been reported in the literature. The following is a description of successful arthroscopic excision of synovial chondromatosis around the proximal tibiofibular joint area in a 14-year-old girl. The study was approved by our institutional review board.

\section{Case details}

A 14-year-old girl presented with right knee pain that had 
persisted for two years. She complained of intermittent pain with flexion or extension. There was no trauma history. The range of motion was fully available. The mechanical tests such as McMurray and full-flexion were positive. No instability was found. Pain radiographs demonstrated the presence of multiple oval calcific lesions in the anterolateral, posteromedial, posterolateral and proximal tibiofibular joint area (Figure 1). Magnetic resonance image (MRI) demonstrated an additional calcification at the posterior septum and multiple small calcifications in the cystic lesion which was located around the popliteus muscle from the proximal area of fibular head to the tibial attachment area of popliteus muscle (Figure 2).

Arthroscopic examination was performed using standard portals to confirm preoperative MRI findings. Posteromedial and posterolateral portals were made under the guidance of the arthroscope introduced through the intercondylar notch, digital palpation and needling. The trans-septal portal was also created to locate and retrieve the calcification at the posterior septum. The small ovoid loose bodies around the proximal tibiofibular joint were also removed through popliteal hiatus using probe and suction (Figure 3). The popliteal hiatus was slightly loose but lateral meniscus was not unstable. Therefore, meniscal repair was not performed. All of the loose bodies were removed and synovectomy was not performed. Histological examination revealed synovial chondromatosis postoperatively (Figure 4).

Rehabilitation for range of motion and ambulation were commenced from the first postoperative day. At the 38months follow-up, the patient had a full range of knee motion and showed no evidence of recurrence (Figure 5).

\section{Discussion}

The knee joint is the most common site of intra-articular synovial chondromatosis. The localized form usually develops in the anterior compartment of knee joint. Until the present time, only three cases of localized synovial chondromatosis around the popliteus muscle were reported. $^{4,5,7}$ Giancane et al. reported a case of synovial chondromatosis in the popliteal-fossa. ${ }^{7}$ They performed only arthroscopic synovectomy to reduce the swelling and local pain without removing the loose bodies. Batheja et al. reported another case of synovial chondromatosis which was formed in the proximal tibiofibular joint and they removed the loose bodies by open debridement. ${ }^{5}$ Bozkurt et al. also reported a case of synovial chondromatosis in four compartments of the knee including proximal tibiofibular joint. ${ }^{4}$ They performed arthrotomy through a medial parapatellar incision to retrieve the loose bodies. And then they performed additional dissection through the lateral portion of the proximal tibia to remove residual loose bodies in the inferior recess of the proximal tibiofibular joint.

In the present case, removing the loose bodies around the popliteal muscle and the proximal tibiofibular joint was performed successfully using only arthroscopic approach. To our knowledge, arthroscopic treatment of synovial chondromatosis of the proximal tibiofibular joint has not been reported in the literature. Bozkurt et al. reported in their cadaveric and MRI study that there is a clear communication between the proximal tibiofibular joint and the knee joint. ${ }^{10}$ The present case illustrates that retrieving the loose bodies was available through the popliteal hiatus and seems to be in accord with the previous study.

It is usually known that the direct approach from the knee joint to the proximal tibiofibular joint using arthroscope is impracticable. One of the reasons why the authors of the past three cases of localized synovial chondromatosis around the popliteus muscle did not perform the arthroscopic management would be that they did not consider the migration of the loose bodies through the popliteal hiatus. In other words, in cases where multiple calcifications are showed around the extra-articular area such as popliteus muscle or proximal tibiofibular joint in plain radiographs or $\mathrm{MRI}$, further arthroscopic examination could be considered to prevent the problems caused by possible migration of the loose bodies to the intra-articular area.

Furthermore, considering the risk of the popliteal artery rupture or the peroneal nerve injury with arthroscopic surgery is lower than that with open debridement or athrotomy, arthroscopic approach should be considered in the treatment for synovial chondromatosis around the popliteal muscle and the proximal tibiofibular joint.

Treatment for synovial chondromatosis is removal of loose bodies with or without synovectomy. It has been debated whether synovectomy is effective for preventing recurrence of synovial chondromatosis. ${ }^{3,11,12}$ Milgram reported in the histopathologic study that synovectomy with removal of loose bodies is necessary for free loose bodies with active intrasynovial proliferation, whereas removal of loose bodies alone is recommended for free osteochondral bodies with no demonstrable intrasynovial disease. ${ }^{11}$ Maurice et al. reported in their study of 43 patients with synovial chondromatosis that recurrence was seen only in cases of 
generalized loose bodies with active synovial lesion. ${ }^{10}$ Furthermore, they also reported that the recurrence rate after operation was same after either synovectomy or simple removal of loose bodies. On the basis of these respects, additional synovectomy was not performed in the present case because active synovial proliferation was not observed in any compartment of joint. Histopathologic examination also revealed no intrasynovial involvement postoperatively. During the 38-months follow-up period, the patient has not showed any evidence of recurrence.

\section{Conclusion}

This case report illustrates that complete removing of synovial chondromatosis located in the extra-articular area of popliteus muscle is available with only arthroscopic approach through a passage between the popliteal hiatus and the proximal tibiofibular joint.

\section{References}

1. Apte SS, Athanasou NA. An immunohistological study of cartilage and synovium in primary synovial chondromatosis. J Pathol. 1992;166(3):277-81. https://doi.org/10.1002/path.1711660310

2. lyengar J, Luke A, Ma CB. An unusual presentation of synovial chondromatosis of the knee: a case report. Clin J Sport Med. 2007;17(2):157-9. https://doi.org/10.1097/JSM.0b013e31803202db

3. Maurice $\mathrm{H}$, Crone M, Watt I. Synovial chondromatosis. J Bone Joint Surg Br. 1998;70(5):807-11.

4. Bozkurt $M$, Uğurlu $M$, Doğan $M$, et al. Synovial chondromatosis of four compartments of the knee: medial and lateral tibiofemoral spaces, patellofemoral joint and proximal tibiofibular joint. Knee Surg Sports Traumatol Arthrosc. 2007;15(6):753-5. https://doi.org/10.1007/s00167-006-0249-9

5. Batheja NO, Wang BY, Springfield D, et al. Fine-needle aspiration diagnosis of synovial chondromatosis of the tibiofibular joint. Ann Diagn Pathol. 2000;4(2):77-80. https://doi.org/10.1016/S1092-9134(00)90015-7

6. Chou PH, Huang TF, Lin SC, et al. Synovial chondromatosis presented as knocking sensation of the knee in a 14-year-old girl. Arch Orthop Trauma Surg. 2007;127(4):293-7. https://doi.org/10.1007/s00402006-0144-3

7. Giancane G, Tanturri de Horatio L, Buonuomo PS, et al. Swollen knee due to primary synovial chondromatosis in pediatrics: a rare and possibly misdiagnosed condition. Rheumatol Int. 2013;33(8):2183-5. https://doi.org/10.1007/s00296-012-2382-8

8. Jesalpura JP, Chung HW, Patnaik S, et al. Arthroscopic treatment of localized synovial chondromatosis of the posterior knee joint. Orthopedics 2010;33(1):49. https://doi.org/10.3928/01477447-20091124-22

9. Kyung BS, Lee SH, Han SB, et al. Arthroscopic treatment of synovial chondromatosis at the knee posterior septum using a trans-septal approach: Report of two cases. Knee 2012;19(5):732-5.

https://doi.org/10.1016/j.knee.2011.10.003

10. Bozkurt M, Yilmaz E, Atlihan D, et al. The proximal tibiofibular joint: an anatomic study. Clin Orthop Relat Res. 2003;406:136-40.

11. Milgram JW. Synovial osteochondromatosis: a histopathological study of thirty cases. J Bone Joint Surg Am. 1977;59(6):792-801. https://journals.Iww.com/jbjsjournal/toc/1977/59060

12. Ogilvie-Harris DJ, Saleh K. Generalized synovial chondromatosis of the knee: a comparison of removal of the loose bodies alone with arthroscopic synovectomy. Arthroscopy 1994;10(2):166-70. https://doi.org/10.1016/S0749-8063(05)80088-X

\section{PEER REVIEW}

Not commissioned. Externally peer reviewed.

\section{CONFLICTS OF INTEREST}

The authors declare that they have no competing interests.

\section{FUNDING}

None

\section{PATIENT CONSENT}

The authors, $L E E, S J$, declare that:

1. They have obtained written, informed consent for the publication of the details relating to the patient(s) in this report.

2. All possible steps have been taken to safeguard the identity of the patient(s).

3. This submission is compliant with the requirements of local research ethics committees. 
Figure 1: Preoperative anteroposterior (a) and lateral (b) radiographs of the right knee showing multiple calcifications in the anterolateral, posteromedial, posterolateral and proximal tibiofibular joint area

(a)

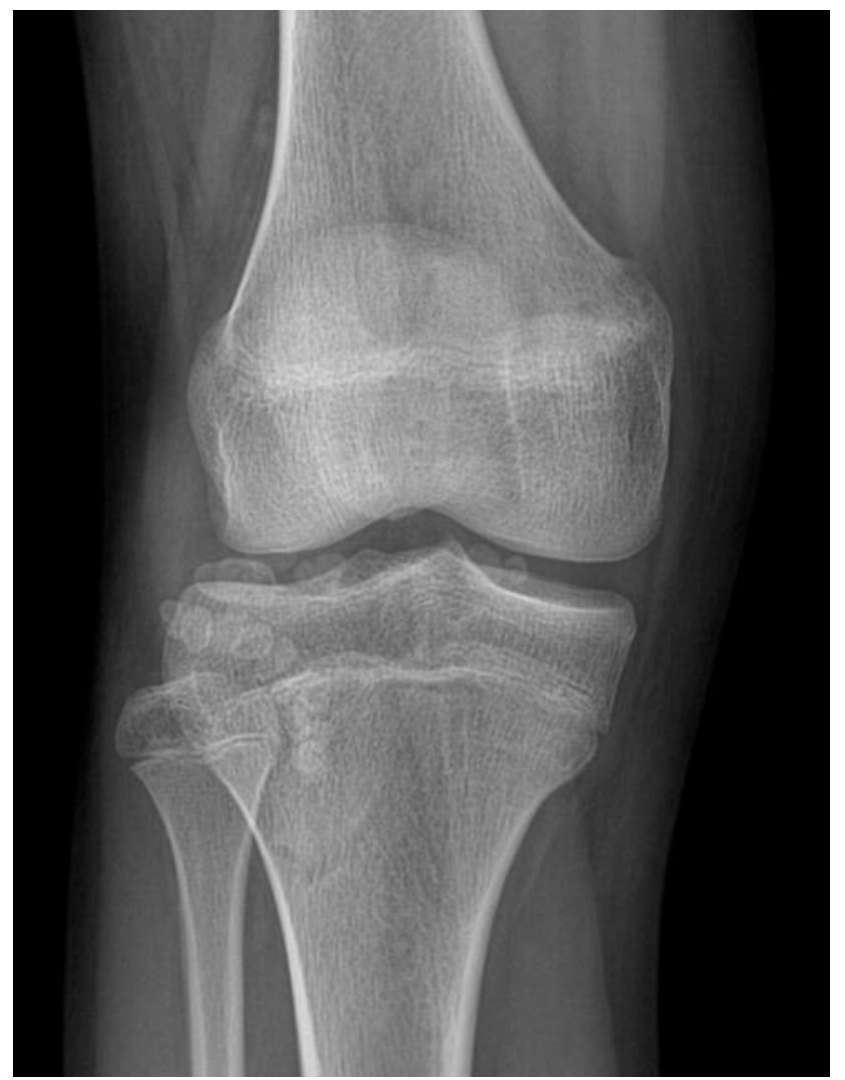

(b)

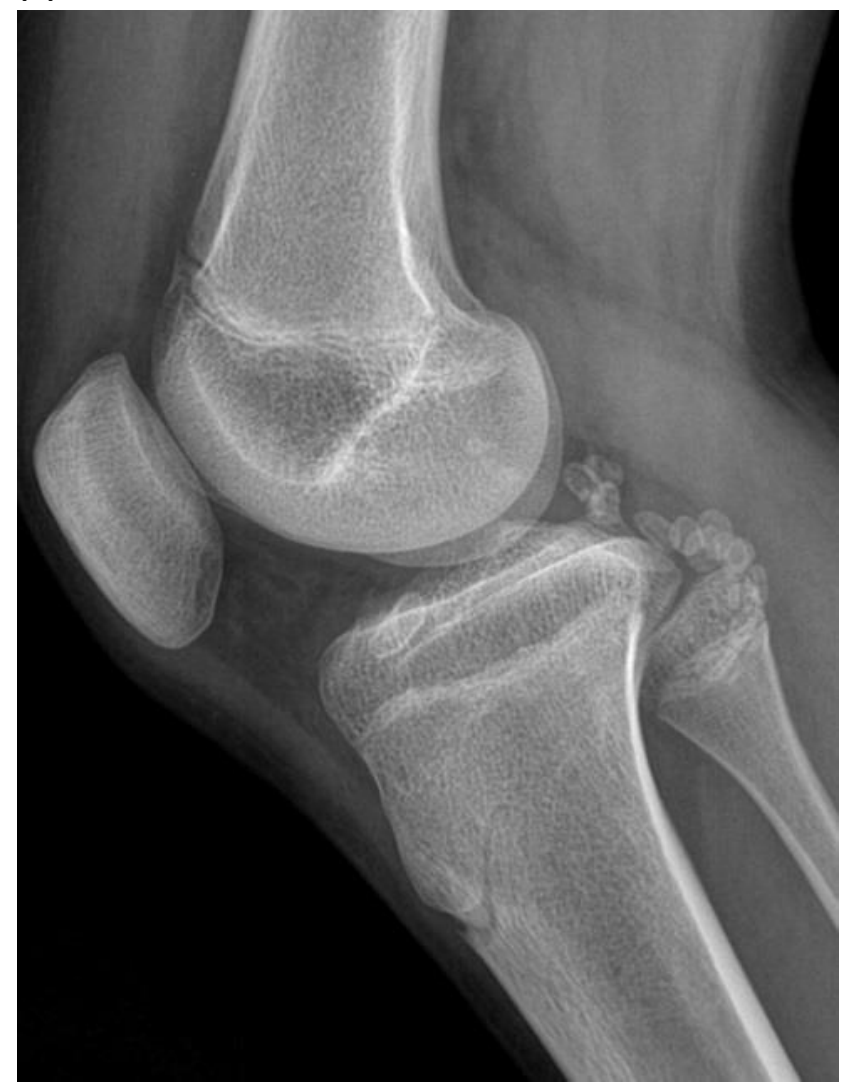

Figure 2: T2-weighted coronal (a) and sagittal (b) MRIs showing multiple calcifications around the popliteus muscle from the proximal area of fibula head to the tibial attachment area of popliteus muscle

(a)

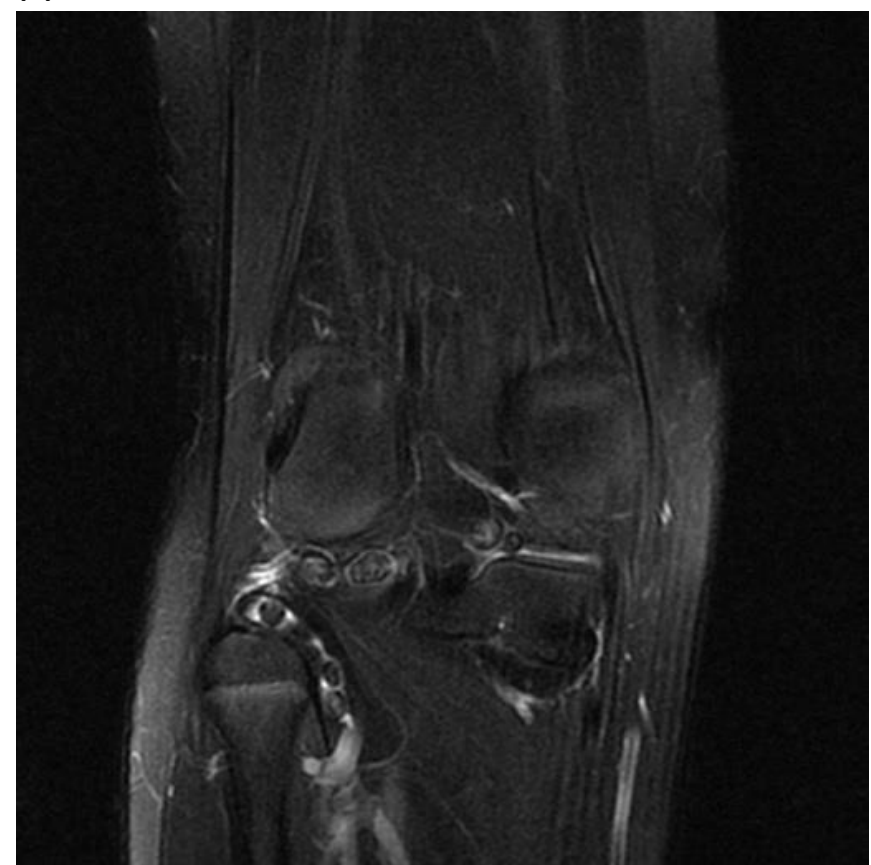

(b)

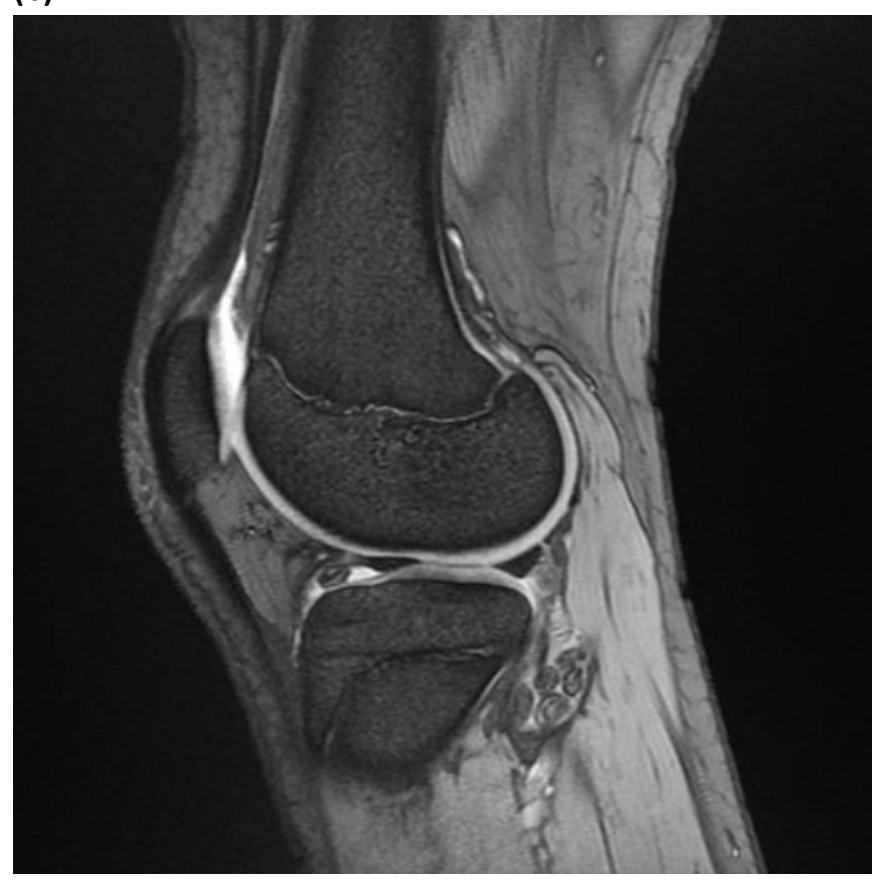


Figure 3: Arthroscopic anterolateral view showing (a) a loose body underneath the lateral meniscus and (b) removal of loose bodies with probing through the popliteal hiatus

(a)

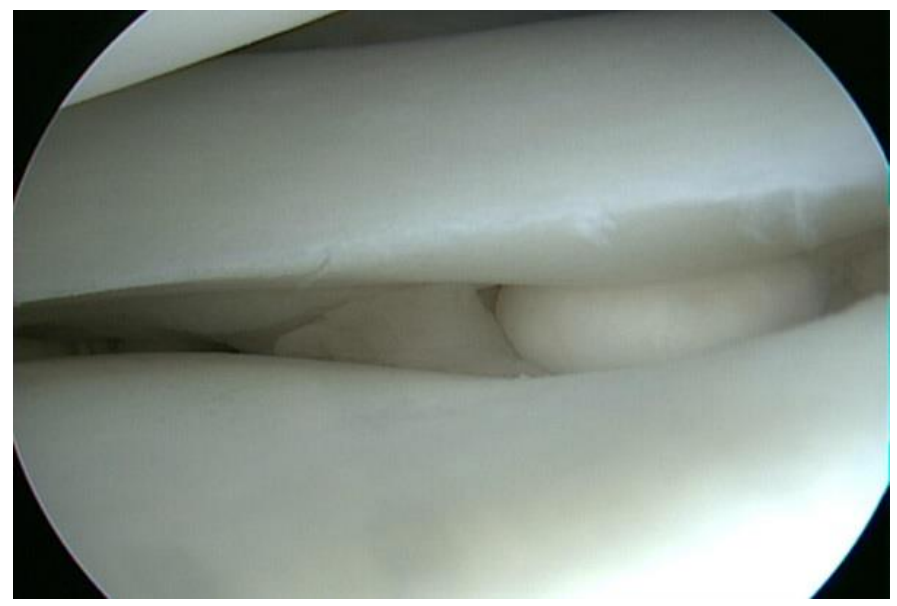

(b)

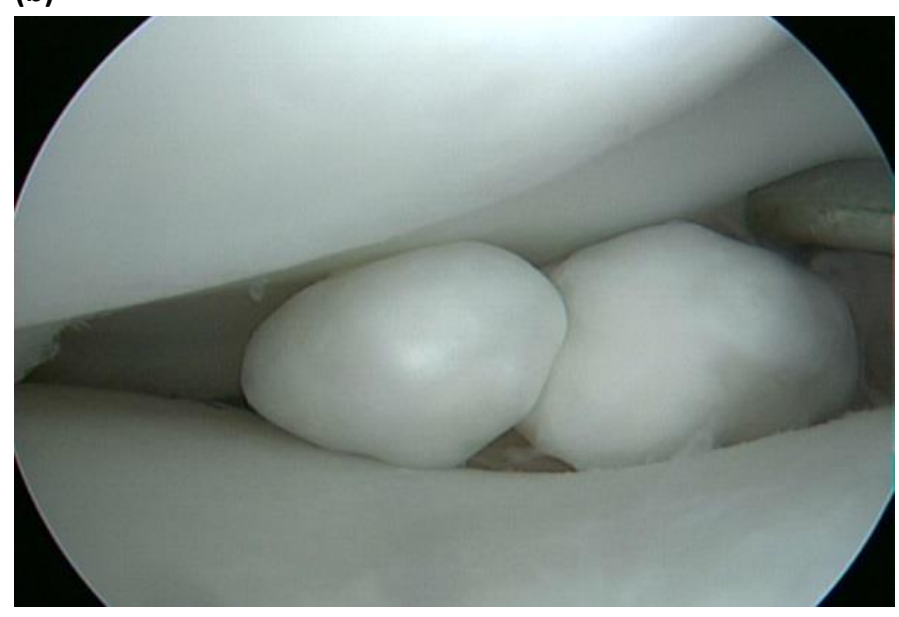

Figure 4: Histopathology showing hyaline cartilage filled with chondrocytes

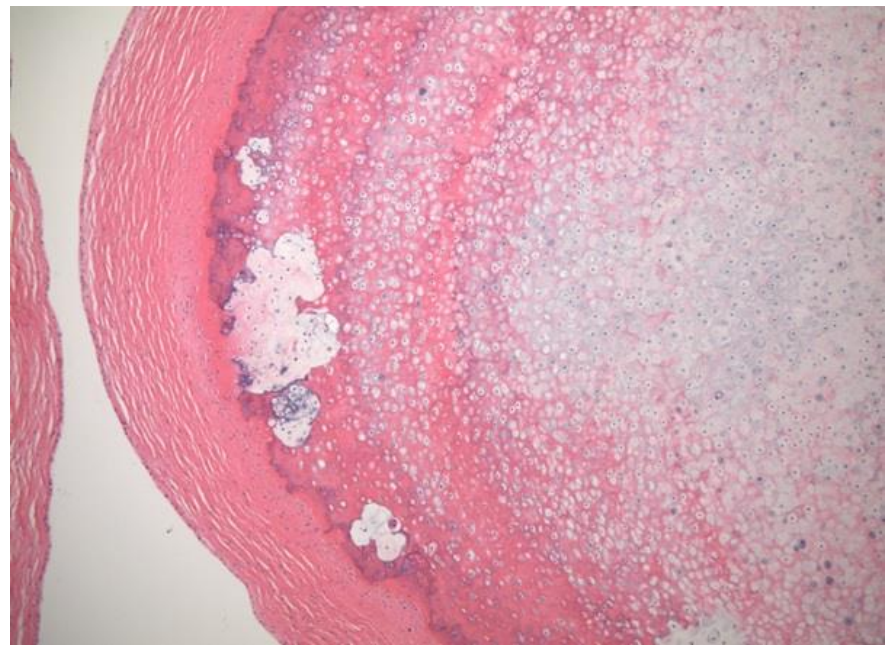

Figure 5: Lateral radiograph of the knee at the final followup showing no recurrence

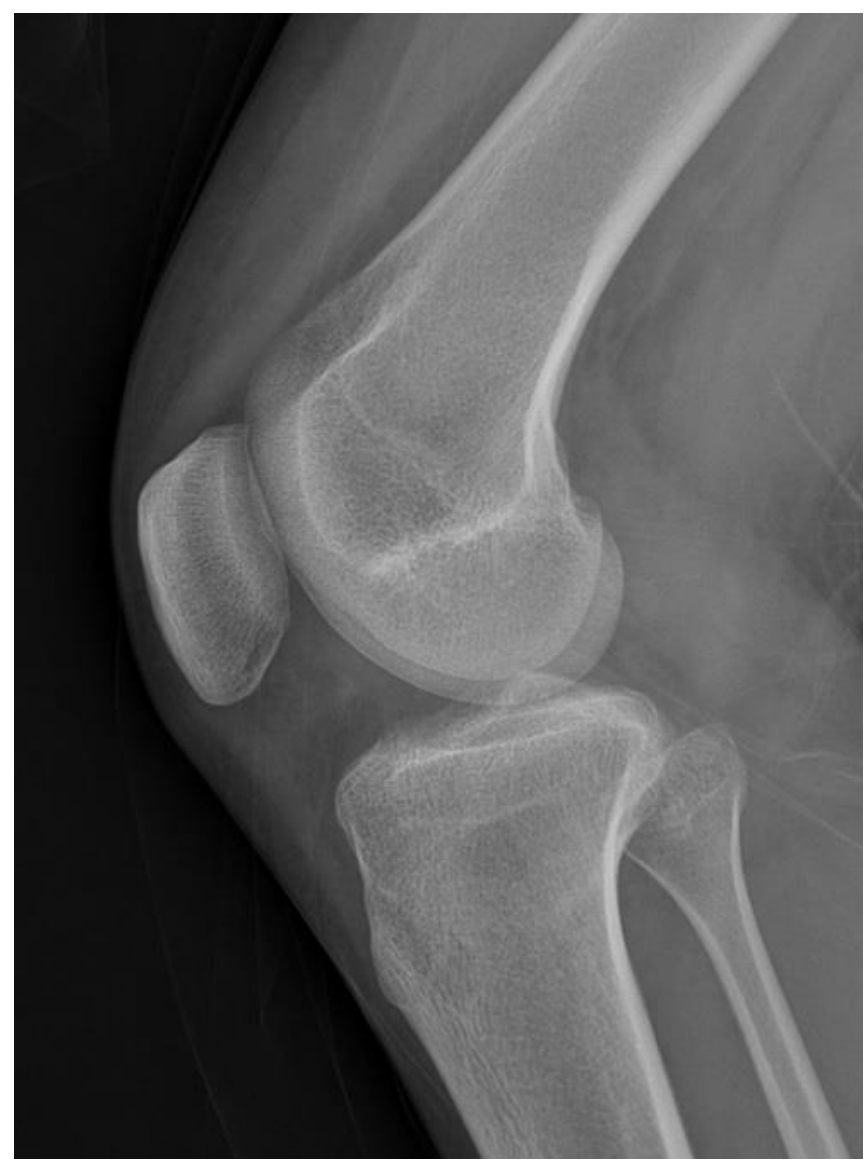

